\title{
The Ninth Asia Pacific Bioinformatics Conference (APBC2011)
}

\author{
Yi-Ping Phoebe Chen ${ }^{1 *}$, Kwang-Hyun $\mathrm{Cho}^{2}$ \\ From The Ninth Asia Pacific Bioinformatics Conference (APBC 2011) \\ Inchon, Korea. 11-14 January 2011
}

The Asia Pacific Bioinformatics Conference (APBC) is a leading conference in the Bioinformatics community and has grown rapidly since its inception in 2003. The goal of the annual conference series is to enable high quality interaction on bioinformatics research.

The past APBC conferences were held in:

1. APBC2003 4-7 Feb 2003: Adelaide Australia

2. APBC2004 18-22 Jan 2004: Dunedin, New Zealand

3. APBC2005 17-21 Jan 2005: Singapore

4. APBC2006 13-16 Feb, 2006: Taipei Taiwan

5. APBC2007 15-17 Jan, 2007: Hong Kong

6. APBC2008 14-17 Jan, 2008: Kyoto Japan

7. APBC2009 13-16 Jan, 2009: Beijing China

8. APBC2010 18-21 Jan, 2010: Bangalore India

The Ninth Asia Pacific Bioinformatics Conference (APBC2011) was held in Incheon, South Korea, the first time in this dynamic country. The conference spanning the dates of the $11^{\text {th }}$ to the $14^{\text {th }}$ of January brought together more than 300 researchers, professional, industry leaders and students from all over the globe. The participants came from institutions in the following 19 countries and regions (in alphabetical order): Australia, Bangladesh, Belgium, Brazil, Canada, China, Germany, India, Italy, Poland, Portugal, Saudi Arabia, Singapore, South Korea, Switzerland, Taiwan, Thailand, UK and USA. The conference program included 6 keynote speakers (Drs. Steven Jones, Luonan Chen, Peer Bork, Sang Yup Lee, Hong Gil Nam and Kenta Nakai), 55 selected talks, 8 tutorials and more than 118 posters.

The titles of the keynote talks are:

- Steven Jones, "Bioinformatics and Cancer Genomics"

- Luonan Chen, "Modeling and Analyzing Nonlinear Biomolecular Networks"
- Peer Bork, "Systemic analysis of the human gut: connecting chemicals, proteins, cells, communities and phenotypes"

- Sang Yup Lee, "Systems metabolic engineering"

- Hong Gil Nam, "Understanding and controlling plant growth and development: Genetic, Systems, and chemical genomic approaches"

- Kenta Nakai, "Information for Transcriptional Regulation"

The tutorial topics for APBC2011 included WebLab: $a$ web-based bioinformatics platform (Jingchu Luo), Promenade through the web programming for biological research using Perl language (Kyung-Hoon Kwon), Bioinformatics analysis of genome $\mathcal{E}$ exome by next generation sequencing (Namshim Kim), Probabilistic Models for Multiple Motif Discovery (Jong Kyoung), Molecular Modeling: Analysis of Protein Structure and Function (Jinhyuk Lee), OASIS: Traditional Korean Medicine Information Portal (Sang-Jun Yea), Information Retrieval and Text Mining Opportunities in Bioinformatics (Jeyakumar Natarajan) and Bioworks: Bioinformation Analysis Pipeline (Seungyoon Nam and Youngmahn Hahn).

The emphasis of APBC has been algorithmic development and innovation in Bioinformatics and this year that theme continued. Reflecting the ever-changing nature of Bioinformatics and is adaption to advances in technology, for the first time, a session was held on next-generation sequencing. A wide range of topics in Bioinformatics were covered at the conference and were categorised as:

- Phylogenetics and Evolution

- Molecular binding and modelling

- Genome Sequencing and Assembly

- Network and system biology

- Structural Bioinformatics

- Pathway analysis

- Proteomics 
- Pattern Recognition and Statistical Inference

- Structure and data modelling

- Translational bioinformatics

- Bioinformatics databases and applications

In 2011, APBC received a substantial number of submissions from a diverse range of countries from Belgium to Brazil. Participants in the 2011 APBC came from every continent with the exception of Africa, however actual participants may represent geographies outside of their institutions. Submissions have come from institutions in a wide range of countries including (alphabetically): Australia, Bangladesh, Belgium, Brazil, Canada, China, Germany, India, Italy, Poland, Portugal, Saudi Arabia, Singapore, South Korea, Switzerland, Taiwan, Thailand, UK and USA.

The APBC2011 conference has received 164 submissions covering a wide range of topics in bioinformatics. The conferences' scope was wide in order to adapt itself quickly to this progressive research field. The broad composition of the expert program committee was well equipped to handle the diversity of topics under the banner of bioinformatics and computational biology. Of the 164 submitted papers, 153 received two or more peer reviews with an acceptance rate of $33 \%$. We wish to thank and acknowledge the Program Committee members and their contributions. The Program Committee members are:

- Tatsuya Akutsu, Kyoto University, JP,

- Masanori Arita, University of Tokyo, JP,

- Joel Bader, Johns Hopkins Uni School of Med, US,

- Alvis Brazma, European Bioinformatics Institute, UK,

- Vladimir Brusic, Dana-Farber Cancer Institute, US,

- Kun-Mao Chao, National Taiwan University, TW,

- Jake Yue Chen, Indiana U. School of Informatics, USA,

- Phoebe Chen, La Trobe University, AU,

- Francis Chin, The University of Hong Kong, HK,

- Jung Kyoon Choi, KAIST, KR,

- In-Sun Choo, KOBIC, KR,

- Kyungsook Han, INHA University, KR,

- Wen-Lian Hsu, Academia Sinica, TW,

- Ju Han Kim, Seoul National University, KR,

- Hsien-Da Huang, National Chiao Tung Uni, TW,

- Daniel Huson, University at Tubingen, Tubingen,

- Daehee Hwang, Postec, KR,

- Jenn-Kang Hwang, National Chiao Tung Uni, TW,

- Tao Jiang, "University of California, Riverside", US,

- Jun Huan, U. of Kansas, USA,

- Young Kim, KRIBB, KR,

- Hyunju Lee, GIST, KR,

- Insuk Lee, Yonsei University, KR,

- Sang Yup Lee, KAIST, KR,

- Ming Li, University of Waterloo, CA,
- Wentian Li, Feinstein Inst for Medical Research, USA,

- Jinyan Li, Institute for Infocomm Research, SG,

- Jingchu Luo, Peking University, CN,

- Bin Ma, University of Waterloo, CN,

- Hiroshi Mamitsuka, Kyoto University, JP,

- Satoru Miyano, University of Tokyo, JP,

- Shinichi Morishita, University of Tokyo, JP,

- Kenta Nakai, University of Tokyo, JP,

- Laxmi Parida, IBM T J Watson Research Center, USA,

- Mark Ragan, University of Queensland, AU,

- Naren Ramakrishnan, Virginia Tech, USA

- Cenk Sahinalp, Simon Fraser University, CA,

- Yasubumi Sakakibara, Keio University, JP,

- David Sankoff, University of Ottawa, CA,

- Thomas Schlitt, King's College London, UK,

- Narayanaswamy Srinivasan, IISc, India,

- Alfonso Valencia, Centro Nacional de Biotech, ES,

- Dianhui Wang, La Trobe University, AU,

- Lusheng Wang, The City Uni of Hong Kong, HK,

- Limsoon Wong, National University of Singapore, SG,

- Hong Yan, City University of Hong Kong, HK,

- Ueng Cheng Yang, National Yang Ming Uni, TW,

- S.M. Yiu, The University of Hong Kong, HK,

- Xuegong Zhang, Tsinghua University, CN,

- Louxin Zhang, National University of Singapore, SG,

- Hongyu Zhao, Yale school of public health, USA.

We would also like to thank all local Organizing Committee members behind the scenes, especially Young Kim, Keun Woo Lee, Kyungsook Han, Ungsik Yu and Micky for their hard work on the website, registration and many other miscellaneous tasks. We also wish to thank Laxmi Parida, Kyung-Hoon Kwon and Sanghyuk Lee for organizing the tutorial session. We also thank Publication \& Web Chair Scott Mann and Sun Shim Choi. For their help we thank industry track chairs Yang Seok Kim and Kap-Seok Yang. We lastly thank The Korean Society for Bioinformatics and Systems Biology, Korea Institute for Science and Technology Information, Korea Research Institute of Bioscience and Biotechnology and The Korean Bioinformation Center for their support.

\footnotetext{
Acknowledgements

This article has been published as part of BMC Bioinformatics Volume 12 Supplement 1, 2011: Selected articles from the Ninth Asia Pacific Bioinformatics Conference (APBC 2011). The full contents of the supplement are available online at http://www.biomedcentral.com/1471-2105/12? issue $=\mathrm{S} 1$.

Author details

'La Trobe University, Melbourne, Australia. ${ }^{2}$ KAIST, Korea.
} 


\section{Competing interests}

The authors declare that they have no competing interests.

Published: 3 March 2011

doi:10.1186/1471-2105-12-S1-I1

Cite this article as: Chen and Cho: The Ninth Asia Pacific Bioinformatics Conference (APBC2011). BMC Bioinformatics 2011 12(Suppl 1):11.

Submit your next manuscript to BioMed Central and take full advantage of:

- Convenient online submission

- Thorough peer review

- No space constraints or color figure charges

- Immediate publication on acceptance

- Inclusion in PubMed, CAS, Scopus and Google Scholar

- Research which is freely available for redistribution 